\title{
Canonical quantum gravity in the Vassiliev invariants arena: II. Constraints, habitats and consistency of the constraint algebra
}

\author{
Cayetano Di Bartolo ${ }^{1}$ Rodolfo Gambini ${ }^{2}$, Jorge Griego ${ }^{2}$, Jorge Pullin ${ }^{3}$ \\ 1. Departamento de Física, Universidad Simón Bolívar, \\ Aptdo. 89000, Caracas 1080-A, Venezuela. \\ 2. Instituto de Física, Facultad de Ciencias, Iguá 4225, esq. Mataojo, Montevideo, Uruguay. \\ 3. Center for Gravitational Physics and Geometry, Department of Physics, \\ The Pennsylvania State University, 104 Davey Lab, University Park, PA 16802.
}

\begin{abstract}
In a companion paper we introduced a kinematical arena for the discussion of the constraints of canonical quantum gravity in the spin network representation based on Vassiliev invariants. In this paper we introduce the Hamiltonian constraint, extend the space of states to non-diffeomorphism invariant "habitats" and check that the off-shell quantum constraint commutator algebra reproduces the classical Poisson algebra of constraints of general relativity without anomalies. One can therefore consider the resulting set of constraints and space of states as a consistent theory of canonical quantum gravity.
\end{abstract}

\section{INTRODUCTION}

\section{A. Preliminaries}

In our previous paper [1], we extended the notion of Vassiliev invariant and other invariants associated with ChernSimons theory to the context of spin networks [2]. We showed that these invariants are loop differentiable in the sense of distributions and we defined an infinitesimal generator of diffeomorphisms in terms of the loop derivative. The generator correctly annihilates diffeomorphism invariant states. In this paper we will introduce a Hamiltonian constraint based on the loop derivative. We will limit the discussion to invariants of trivalent vertices and will only concentrate on the "Euclidean" part of the Hamiltonian constraint in the sense of Barbero [3] and Thiemann [1]. Limiting ourselves to trivalent intersections is clearly unphysical since the volume operator identically vanishes on such states. However, the essence of most calculations is already present with trivalent intersections and the calculational difficulty is significantly lower than with four or higher valent intersections. We will come back to this issue in the discussion section where we will highlight which results are quite plausibly going to survive when one goes to higher valent intersections. We will then extend the space of invariants through the construction of "habitats" such that the resulting states are not diffeomorphism invariant. Based on these states we will check the consistency of the quantum commutator algebra of diffeomorphisms and Hamiltonian. We will show that there are no anomalies, therefore constituting a consistent theory of canonical quantum gravity.

\section{B. Strategy}

We will start in the next section by introducing the Hamiltonian constraint of quantum gravity in terms of the loop derivative. This derivation will be "generic" in the sense that we will motivate it through formal manipulations of the loop transform and therefore will not make specific assumptions about the quantum state in the connection representation on which the operator is acting upon. Without being more precise about the space of states upon which it acts, we cannot guarantee that the operator is well defined. We will show that on several spaces related to the Vassiliev invariants the operator is indeed well defined. The results obtained will be later applied in specific "habitats" in which we will show that the operators exist and we can compute correctly the commutators. This we will discuss in section III. The need to introduce new (non-diffeomorphism-invariant) "habitats" is based on the fact that the spaces we have considered in the companion paper are all diffeomorphism invariant and therefore one cannot explore in a non-trivial way commutators involving the diffeomorphisms. We will introduce an explicit example of such a habitat in detail, the "functions with marked vertices". In the end we will see that we will be able to recover the classical Poisson algebra at the level of quantum commutators, but there will be subtle issues involved in the definition of the right hand side of the commutator of two Hamiltonians, which we will discuss in detail. The last section will be devoted to discussing the implications of the level of consistency achieved by the theory. 


\section{THE HAMILTONIAN CONSTRAINT}

We wish to introduce a quantum version of the Hamiltonian constraint of canonical general relativity. Following Thiemann [4, one can construct this operator introducing the real version of the Ashtekar variables first discussed by Barbero [3]. In this formulation, the canonical pair consists of a set of (densitized) triads $\tilde{E}_{i}^{a}$ and as conjugate variables real-valued $S U(2)$ connections $A_{a}^{i}$. The Hamiltonian constraint of canonical, real, Lorentzian general relativity in this framework consists of two terms,

$$
H(N)=H_{E}(N)+\int d^{3} x N(x) \frac{4}{G^{3}}\left\{A_{a}^{i}(x), K\right\}\left\{A_{b}^{j}(x), K\right\}\left\{A_{c}^{k}(x), V\right\} \epsilon_{i j k} \tilde{\epsilon}^{a b c},
$$

where

$$
\begin{aligned}
H_{E}(N) & =\int d^{3} x N(x) \frac{2}{G}\left\{A_{a}^{i}(x), V\right\} F_{b c}^{i}(x) \tilde{\epsilon}^{a b c}, \\
K & =-\frac{1}{G}\left\{V, H_{E}(1)\right\}, \\
V & =\int d^{3} x \sqrt{\underset{\epsilon}{\sim} a b c \tilde{E}_{i}^{a}(x) \tilde{E}_{j}^{b}(x) \tilde{E}_{k}^{c}(x) \epsilon^{i j k}} .
\end{aligned}
$$

In these expressions, $G$ is the Newton constant and in (3) $H_{E}(1)$ means that the smearing function $N(x)$ is unity. This remarkable formulation implies that the Hamiltonian constraint is composed by two terms. The first one, $H_{E}$, coincides with the Hamiltonian constraint one would obtain in a canonical formulation of general relativity on a manifold of Euclidean signature. The Lorentzian theory is therefore attained through the addition of an extra term. Both terms are given as expressions involving the Poisson bracket of a connection with the volume of the space $V$ and with the function $K$. The latter can be in turn obtained as a Poisson bracket of the Euclidean part of the Hamiltonian constraint with the volume.

In this paper we will only concentrate on realizing at a quantum mechanical level the Euclidean part of the Hamiltonian. If the reader wishes, we are considering canonical quantum gravity in the Euclidean context. However, it should be noticed that the additional term in the Hamiltonian is obtained by successive commutators of the operator we will consider, with the volume operator. This suggests that one could generalize our construction to the Lorentzian case rather straightforwardly, but this has not been studied in detail. The work of Thiemann also shows that similar structures arise if one couples the theory to matter. We will in this paper only concentrate on the vacuum case but again one does not see a priori impediments to extend our treatment to general relativity coupled to matter.

To implement the Hamiltonian constraint we will partially use the same procedure proposed by Thiemann. We will not describe it again in full detail here, we just list some of the salient features. One starts by writing the classical expression (2) as

$$
H(N)=\frac{2}{G} \lim _{\epsilon \rightarrow 0} \int d^{3} x \int d^{3} y N(y) \epsilon^{a b c} \operatorname{Tr}\left[F_{a b}(y)\left\{A_{c}(x), V\right\}\right] f_{\epsilon}(y, x)
$$

where $f_{\epsilon}(y, x)$ is a regularization of the Dirac delta function, i.e. $\lim _{\epsilon \rightarrow 0} f_{\epsilon}(y, x)=\delta^{3}(y, x)$. Strictly speaking, this expression is only gauge invariant in the limit, so to preserve gauge invariance in the regularization procedure we will "join" the $F_{a b}$ with the connection using an infinitesimal piece of holonomy $h\left(\pi_{x}^{y}\right)$ along an arbitrary path $\pi$ going from $x$ to $y$, which tends to the null path in the limit $\epsilon \rightarrow 0$. To unclutter the notation we will therefore denote $F_{a b}\left(\pi_{x}^{y}\right) \equiv h\left(\pi_{x}^{y}\right) F_{a b}(y) h\left(\pi_{y}^{x}\right)$

$$
H(N)=\frac{2}{G} \lim _{\epsilon \rightarrow 0} \int d^{3} x \int d^{3} y N(y) \epsilon^{a b c} \operatorname{Tr}\left[F_{a b}\left(\pi_{x}^{y}\right)\left\{A_{c}(x), V\right\}\right] f_{\epsilon}(y, x)
$$

In order to prepare the classical expression for quantization, we triangulate the space-like hypersurface $\Sigma$ in terms of elementary tetrahedra $\Delta$. The triangulation has the following properties: we select a finite set of distinct points of $\Sigma$, denoted as $\{v\}$. At each of these points we choose three independent directions $\left(\hat{u}_{1}, \hat{u}_{2}, \hat{u}_{3}\right)$ and construct the eight tetrahedra with vertex $v$ and edges $\left( \pm u_{1}, \pm u_{2}, \pm u_{3}\right)$, with $u_{i}=\epsilon \hat{u}_{i}$. The eight tetrahedra saturating $v$ define a closed region $\square_{v}$ of length scale $\epsilon$. The remaining region $\Sigma-\cup_{v} \square_{v}$ is triangulated by arbitrary tetrahedra $\Delta^{\prime}$. The motivation for this peculiar discretization is that when we promote the classical expression to a quantum operator, we will adapt the triangulation to the spin network of the state in question by choosing the points $v$ to coincide with the vertices of the spin network. In terms of this triangulation of space we can write equation (6) in the form, 


$$
H(N)=\frac{2}{G} \lim _{\diamond \rightarrow 0} \int d^{3} y \sum_{\diamond \in\left\{\square_{v}, \Delta^{\prime}\right\}} \mathcal{V}_{\diamond} N(y) \epsilon^{a b c} \operatorname{Tr}\left[F_{a b}\left(\pi_{v_{\diamond}}^{y}\right)\left\{A_{c}\left(v_{\diamond}\right), V\right\}\right] f_{\diamond}(y),
$$

where the regions $\diamond$ indicate either a box $\square_{v}$ or a tetrahedron $\Delta^{\prime}$, and $v_{\diamond}$ is any point interior to $\diamond$. Conventionally we will choose for the boxes $v_{\square_{v}}=v$, and for the tetrahedra $v_{\Delta^{\prime}}$ will represent one of its vertices. To discretize the integral we have introduced the volume of each region $\mathcal{V}_{\diamond}$,

$$
\mathcal{V}_{\diamond} \epsilon^{a b c}=\frac{\alpha_{\diamond}}{6} \epsilon^{i j k} u_{i}^{a} u_{j}^{b} u_{k}^{c},
$$

where $\alpha_{\diamond}=8$ if $\diamond=\square$, and $\alpha \diamond=1$ if $\diamond=\Delta^{\prime}$. In this last case, the $u_{i}$ 's represent the edges of the tetrahedra $\Delta^{\prime}$ adjacent to $v_{\Delta^{\prime}}$. We also have adapted the regularization of the Dirac delta function to the tetrahedral decomposition by defining,

$$
f_{\diamond}(y)=\frac{\Theta_{\diamond}(y)}{\mathcal{V}_{\diamond}}
$$

where $\Theta_{\diamond}(y)$ is one if $y \in \diamond$ and zero otherwise. We now replace in (7) the $\epsilon^{a b c}$ using formula (8) and we represent $u_{k}^{c} A_{c}\left(v_{\diamond}\right)$ using a holonomy in the fundamental representation along the edge $u_{k}$ of the triangulation,

$$
H(N)=\lim _{\diamond \rightarrow 0} \int d^{3} y \sum_{\diamond \in\left\{\square_{v}, \Delta^{\prime}\right\}} \frac{\alpha_{\diamond}}{3 G} \epsilon_{i j k} u_{i}^{a} u_{j}^{b} N(y) \operatorname{Tr}\left[F_{a b}\left(\pi_{v_{\diamond}}^{y}\right) h\left(u_{k}\right)\left\{h^{-1}\left(u_{k}\right), V\right\}\right] f_{\diamond}(y),
$$

and we retraced the path described by the holonomy to preserve gauge invariance. It should be noticed that this retracing does not contribute to the expression at leading order in $\epsilon$ (the scale parameter of the regions $\diamond$ ). We will now proceed to study the quantization of the last expression. For that we need to "adapt" the triangulation we introduced to a spin network state $s$ 四. Let $\left\{v_{s}\right\}$ be the set of vertices of the spin network and let $\left(e_{1 m}, e_{2 m}, e_{3 m}\right)$ be the triples of non-coplanar edges incident at $v_{s}\left(m=1, \ldots, E\left(v_{s}\right)\right.$, being $E\left(v_{s}\right)$ the number of such triples). To adapt the triangulation to the spin network state we perform two operations: first we identify the points $v$ of the boxes $\square_{v}$ with the vertices $v_{s}$ and second, given a triple of edges $\left(e_{1 m}, e_{2 m}, e_{3 m}\right)$ incident at $v_{s}$, we orientate the three unit vectors $\left(\hat{u}_{1}, \hat{u}_{2}, \hat{u}_{3}\right)$ along the tangents of the edges at $v_{s}$. Then we can write,

$$
\epsilon \hat{u}_{i m}^{a}=\int_{e_{i m}} d w^{a} \Theta_{\square_{v_{s}}}(w) .
$$

To simplify the notation we identify from now on $v_{s} \equiv v$. As in the previous paper, we assume we are acting on a state given by a loop transform,

$$
\Psi(s)=\int D A \Psi[A] W_{A}[s],
$$

and we realize the Hamiltonian operator over the spin network wavefunctions by promoting the classical expression (10) as an operator acting on the Wilson net appearing in the loop transform, very much as we did in the companion paper when we discussed the diffeomorphism operator,

$$
\begin{aligned}
H(N) \Psi(s)= & \frac{8}{3 G} \int D A \Psi(A) \lim _{\square \rightarrow 0} \int d^{3} y \sum_{v \in s} \sum_{m=1}^{E(v)} \frac{\epsilon_{i j k}}{E(v)} \int_{e_{i m}} d w^{a} \Theta_{\square_{v}}(w) \int_{e_{j m}} d w^{b} \Theta_{\square_{v}}(u) \times \\
& \times N(y) \operatorname{Tr}\left(F_{a b}\left(\pi_{v}^{y}\right) h\left(u_{k m}\right)\left[h^{-1}\left(u_{k m}\right), V\right]\right) f_{\square_{v}}(y) W_{A}(s) .
\end{aligned}
$$

A first observation is that the volume operator has non-vanishing contributions only at the vertices of the spin net, so we replace the sum in the Hamiltonian over all $\diamond$ 's by a sum over all the vertices of the spin net. The second observation is that, for an n-valent vertex, we have taken the average over all the non-coplanar triples of edges associated with this vertex. Finally notice that only one of the terms in the commutator contributes, that with the volume operator on the left. The one with the volume operator on the right vanishes since it is proportional to the trace of the Lie algebra element $\operatorname{Tr}\left(F_{a b} h_{u_{k m}} h_{u_{k m}}^{-1}\right)$. Let us now consider a generic $n$-valent vertex and study the action of the operator on the Wilson net. We assume that the holonomies are all outgoing from $v$. Schematically, we are trying to represent the action of $\operatorname{Tr}\left(F_{a b} h_{e} V h_{e}^{-1}\right) W_{A}(s)$, with $e$ a generic edge of spin円 $J$ incident at $v$. An

\footnotetext{
${ }^{1}$ We use capital Latin letters to design the "spins" of the edges of the spin network.
} 
$n$-valent vertex is characterized by an intertwiner that can be represented through a vector $\vec{I}$ of $n-3$ of irreducible representations. The factor $h_{e}^{-1}$ acts by adding a line of spin $1 / 2$ ingoing from the vertex $v$ and parallel to the line $e$,

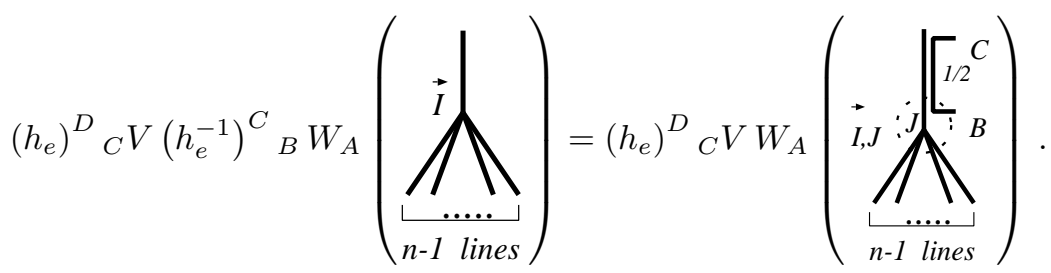

Notice that the insertion of the holonomy leaves a Wilson net that is not gauge invariant anymore, we represent this by keeping the group indices $B, C$ in the diagram.

If one was acting on an $n$-valent vertex characterized by an intertwiner $\vec{I}$, this leaves an $(n+1)$-valent vertex characterized by intertwiners $\vec{I}, J ; J$ being in the diagram the spin of the line connecting the original vertex to the point (infinitesimally nearby) where the holonomy was inserted. Notice that $J$ coincides with the spin of the original line $e$. We denote that the line is infinitesimally close by the dashed circle. We now act with the volume operator. For that we first have to reduce the product of holonomies associated with $e$ to a superposition of irreducible representations. This procedure defines a new intertwiner for the vertex $v$ over which the action of the volume operator is well-defined,

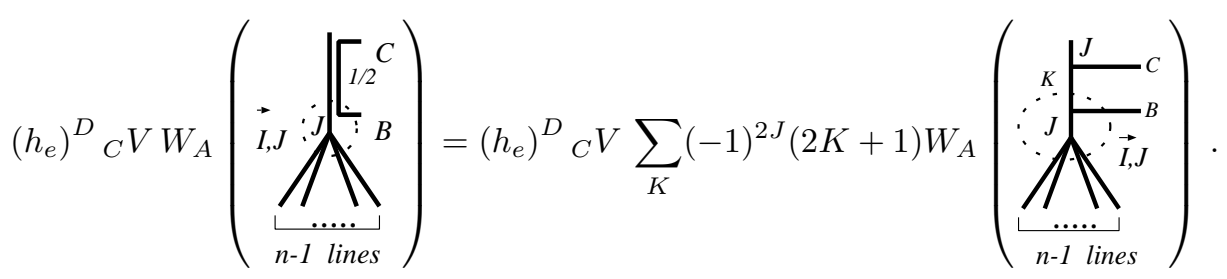

The action of the volume operator can be represented by a matrix that rearranges the intertwining of the vertex. This matrix elements would depend also on the spins of the external edges of the vertex (notice that $K$ is the color of one of this external edges). Schematically,

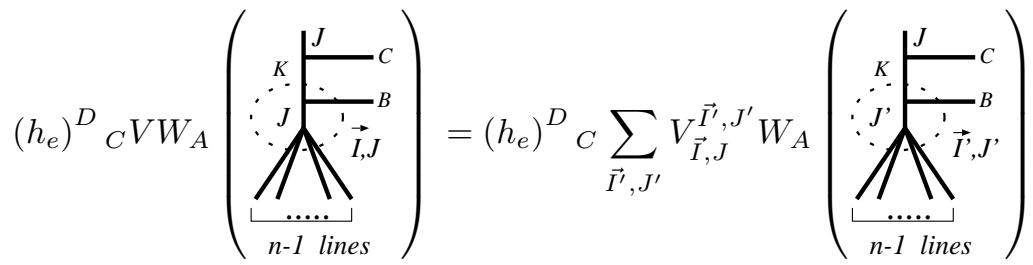

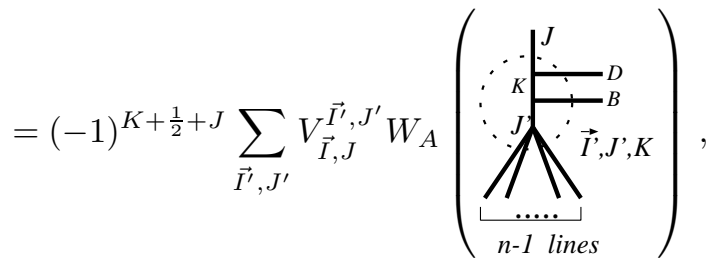

where in the last step we reduce the line of spin $1 / 2$ of the holonomy using recoupling theory. One is left with a double insertion at the vertex, which we can rearrange via recoupling, since everything is happening infinitesimally close to the vertex. We write the result in the following way,

$$
\left(h_{e}\right)^{D}{ }_{C} V\left(h_{e}^{-1}\right)^{C}{ }_{B} W_{A}\left(\left.\right|_{\substack{\vec{I} \ldots \\ n-1 \text { lines }}}\right)
$$




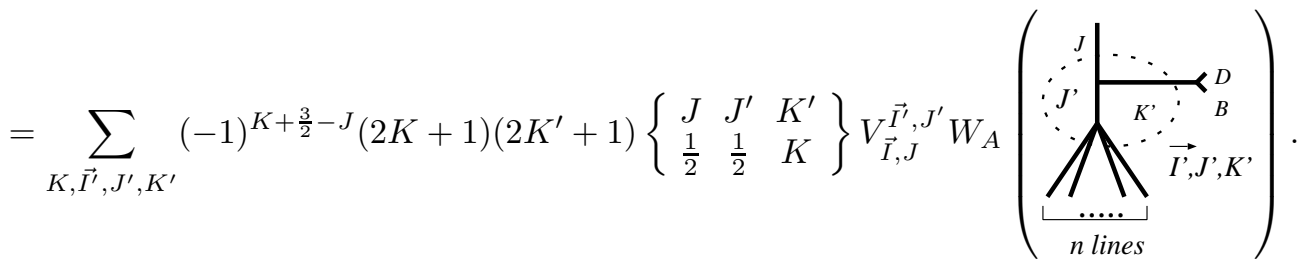

The above expression has now to be contracted with $F_{a b}$ at the open strands labeled with $B$ and $D$. One can generate $F_{a b}$ by introducing an extension of the idea of loop derivative for spin networks. The reason why one does not simply recover the ordinary loop derivative is that the edge before and after the point of the insertion of $F_{a b}$ are in general in different representations $J$ and $J^{\prime}$. The ordinary loop derivative inserts an $F_{a b}$ without changing the representation of the original line. This definition of loop derivative appeared as natural in the context of loops, however in terms of spin networks one expects in general to have a situation like the one we have here. One can use the same set of constructions we did for the ordinary loop derivative of the invariant $E(s, \kappa)$ to compute the action of this generalized loop derivative ${ }^{2}$. However, we will not pursue the exploration of this generalized derivative in this paper. Instead we will concentrate on the case of trivalent intersections. If the spin networks considered have trivalent intersections, when we repeat the construction we did above, the representations of the edges before and after the insertion of $F_{a b}$ are the same. The operator we then obtain to represent $F_{a b}$ corresponds in this case to the ordinary loop derivative. We have now to consider the action of the volume operator over a four-valent vertex with only three real edges [5],

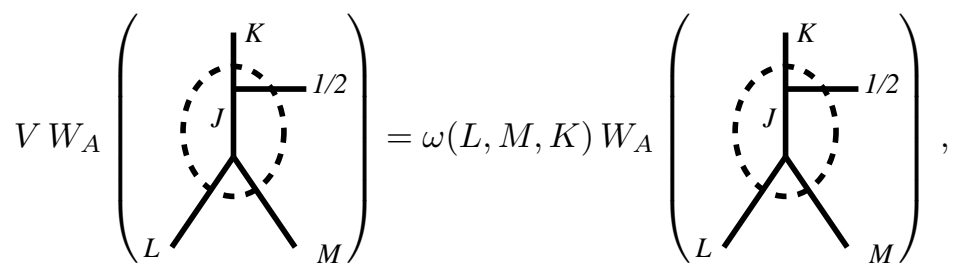

with 3

$$
\omega(L, M, K)=\sqrt{\Lambda_{L M K} \sqrt{K(K+1)}\left|\left\{\begin{array}{ccc}
K & \frac{1}{2} & K+\frac{1}{2} \\
K-\frac{1}{2} & 1 & K
\end{array}\right\}\left\{\begin{array}{ccc}
1 & K-\frac{1}{2} & K+\frac{1}{2} \\
1 & L & L \\
1 & M & M
\end{array}\right\}\right|},
$$

where $\Lambda_{L M K} \equiv \Lambda_{L} \Lambda_{M} \Lambda_{K}$, and $\Lambda_{J}=\sqrt{J(J+1)(2 J+1)}$. Using this result, the matrix elements of equation (18) are reduced to the following simple expression for trivalent vertices $\left(\vec{I}=\vec{I}^{\prime} \equiv 0\right.$ in this case $)$,

$$
V_{J}^{J^{\prime}}(L, M, K)=\delta_{J}^{J^{\prime}} \omega(L, M, K)
$$

where $K=J \pm \frac{1}{2}$, and $L$ and $M$ are the other spins of the edges incident at the trivalent vertex. Therefore the spin of the link going from the intersection to the point of insertion of $F_{a b}$ is unchanged through the action of the volume operator. By inserting the $F_{a b}$ we obtain the final action of the Hamiltonian constraint on trivalent vertices,

$$
H(N) \psi\left(\sum_{e_{3}}^{e_{1}}\right)=\frac{8}{3 G} \lim _{\square \rightarrow 0} \int d^{3} y \sum_{v \in s} \epsilon_{i j k} \int_{e_{i}} d w^{a} \Theta_{\square_{v}}(w) \int_{e_{j}} d t^{b} \Theta_{\square_{v}}(t) N(y) f_{\square_{v}}(y) \times
$$

\footnotetext{
${ }^{2} \kappa$ is proportional to the inverse of the coupling constant of Chern-Simons theory. For a definition of the invariant $E(s, \kappa)$, see the companion paper, section II.C.

${ }^{3}$ The result listed here differs from that given in reference [5], because we are using a different normalization in the definition of spin networks, details can be seen in 19]. Our convention agrees up to a numerical factor with Thiemann's conventions 6 .
} 


$$
\times \rho_{k}\left(\vec{J}_{v}\right) \Delta_{a b}^{(k)}\left(\pi_{v}^{y}\right) \psi\left(\sum_{e_{3}}^{e_{1}}\right) .
$$

The superscript on the loop derivative denotes at which of the incident edges at the intersection it adds the extra strand $\pi$. Notice that since $k$ is summed by the Einstein convention, the derivative ends up acting on all edges (we have abused of the summation convention allowing the index $k$ to be repeated three times in the last expression). The vector $\overrightarrow{J_{v}}=\left(J_{1}, J_{2}, J_{3}\right)$ labels the spins of the tree edges of the spin network incident at $v\left(J_{i}\right.$ is the spin of the edge $\left.e_{i}\right)$, and the group factor $\rho_{k}\left(\overrightarrow{J_{v}}\right)$ is given by the expression,

$$
\rho_{k}\left(\vec{J}_{v}\right):=\sum_{Q}(-1)^{Q+\frac{1}{2}+J_{k}} \sqrt{\frac{3}{2}} \frac{(2 Q+1)}{\Lambda_{J_{k}}}\left\{\begin{array}{ccc}
J_{k} & J_{k} & 1 \\
\frac{1}{2} & \frac{1}{2} & Q
\end{array}\right\} \omega\left(J_{k 1}, J_{k 2}, Q\right),
$$

where the indices $(k, k 1, k 2)$ take cyclic values in the set $(1,2,3)$.

We have therefore a general expression for the Hamiltonian constraint acting on any loop-differentiable wavefunction with support on trivalent spin networks, that bears a relationship with a wavefunction in the connection representation $\Psi(A)$ given by (12).

At this point it is worthwhile comparing this action of the Hamiltonian constraint we just introduced with the original proposal of a Hamiltonian constraint in the loop representation (doubly densitized) in terms of loop derivative as introduced in [7]. Such constraint was written in [8] as,

$$
H(\underset{\sim}{N}) \Psi(\gamma)=\frac{2}{G} \lim _{\epsilon \rightarrow 0} \epsilon \int d^{3} x \int_{\gamma} d y^{a} \int_{\gamma} d z^{b} \underset{\sim}{N}(x) f_{\epsilon}(y, z) \delta^{3}(y-z) \Delta_{a b}\left(\gamma_{o}^{x}\right) O_{y, z} \Psi(\gamma)
$$

The Hamiltonian acted on functions of loops and the operator $O$ had the action of re-routing one of the lobes of the partition of the loop determined by the points $y, z$. We see two fundamental differences with the operator we just introduced. The first one is the re-routing operator $O$. This operator arose in the loop representation to account for the fact that states based on loops in the fundamental representation do not diagonalize operators like the volume. In the spin network context the operator is replaced by the group factors we discussed. More important is the difference concerning density weights. In the Hamiltonian in terms of loops we see that one obtains a doubly densitized quantity by considering the product of a Dirac delta times the regulator. To understand properly this difference, it is worthwhile considering on which spaces of functions are these operators meant to operate upon. In the Hamiltonian in terms of loops one had in mind that the loop derivative was acting on functions of loops such that the result was a smooth function. An example of such functions would be holonomies built with a smooth connection. The resulting expression would then consist of a smooth tensor, $F_{a b}$, a Dirac delta integrated on three dimensional space and, in the limit, a two dimensional Dirac delta given by $\lim _{\epsilon \rightarrow 0} \epsilon f_{\epsilon}(y, z)$ integrated along the two one dimensional integrals. The result is finite but is regularization dependent, since the two dimensional Dirac delta has an inverse power of the three dimensional volume in it that is not compensated.

If we now turn our attention to the Hamiltonian we introduced in this paper, and consider its action on a state of the form $E(s, \kappa)$, we will see a different behavior. Given the action (companion paper: formulas 71 and 44 ) of the loop derivative on $E(s, \kappa)$ we get,

$$
\begin{aligned}
& H(N) E\left(\bigwedge_{e_{2}}^{e_{1}}, \kappa\right)=-\frac{8 \kappa}{3 G} \lim _{\square \rightarrow 0} \int d^{3} y \sum_{v \in s} \epsilon_{i j k} \int_{e_{i}} d w^{a} \Theta_{\square_{v}}(w) \int_{e_{j}} d t^{b} \Theta_{\square_{v}}(t) N(y) f_{\square_{v}}(y) \times \\
& \times \rho_{k}\left(\vec{J}_{v}\right) \epsilon_{a b c} \int_{e_{k}} d z^{c} \delta^{3}(z, y) E\left(\begin{array}{c}
e_{k} \\
e_{k 1}
\end{array} \vdots_{e_{k 2}}, \kappa\right)
\end{aligned}
$$


where the extra edge added by the loop derivative starts and ends in the edge $e_{k}$ and as before, $(k, k 1, k 2)$ take cyclic values in the set $(1,2,3)$. The reason for this is that if it ended in any other edge incident on the vertex one would (in the limit $\square \rightarrow 0$ ) get zero for the action since one would have an integral repeated over one of the edges contracted with $\epsilon_{a b c}$.

The action on $E(s)$ of the loop derivative that appears in the Hamiltonian constraint, for trivalent vertices reduces therefore to a chord diagram. If we evaluate the chord diagram using recoupling identities, we finally get,

$$
\begin{aligned}
& H(N) E\left(\bigwedge_{e_{2}}^{e_{1}}, \kappa\right)=-\frac{8 \kappa}{3 G} \lim _{\square \rightarrow 0} \sum_{v \in s} \int d^{3} y \epsilon_{a b c} \epsilon_{i j k} \int_{e_{i}} d w^{a} \Theta_{\square_{v}}(w) \int_{e_{j}} d t^{b} \Theta_{\square_{v}}(t) \times \\
& \left.\int_{e_{k}} d z^{c} \delta^{3}(z, y) N(y) f_{\square_{v}}(y) \rho_{k}\left(\vec{J}_{v}\right) J_{k}\left(J_{k}+1\right) E\left(\bigwedge_{e_{2}}\right)^{e_{1}}, \kappa\right) .
\end{aligned}
$$

At this point it is worthwhile continuing the comparison with the doubly densitized case. If we evaluate the integrals involved in the above expression we see that we have six one dimensional integrals and two three dimensional Dirac delta functions. In the limit we therefore have a finite result, that is defined independent of the background structures introduced by the regulator $f_{\square}(y)=\Theta_{\square}(y) / \mathcal{V}_{\square}$. One first performs the integral in $y$, which fixes $y=z$ and then one is left with three one dimensional integrals with three step functions (two explicit, one present in the $f_{\square_{v}}$ ). The result of the integrals is $\mathcal{V}_{\Delta}$, which cancels the denominator of the $f_{\square}\left(\mathcal{V}_{\square}=8 \mathcal{V}_{\Delta}\right)$ and one is left with a finite result, that in the limit $\square \rightarrow 0$ gives,

$$
H(N) E\left(\chi_{e_{3}}^{e_{1}}, \kappa\right)=-\frac{\kappa}{G} \sum_{v \in s} N(v) \rho\left(\vec{J}_{v}\right) E\left(\lambda_{e_{3}}^{e_{1}}, \kappa\right),
$$

where

$$
\rho\left(\vec{J}_{v}\right):=4 \sum_{k=1}^{3} J_{k}\left(J_{k}+1\right) \rho_{k}\left(\vec{J}_{v}\right),
$$

is the factor group associated with the vertex $v$.

We have introduced a quantum version of Thiemann's singly-densitized classical Hamiltonian in terms of the loop derivative that has the remarkable following properties:

a) It has a significant resemblance to the original doubly-densitized Hamiltonian proposed in the loop representation [7], which in turn closed the constraint algebra formally [8]. We will discuss the implications of this in the next section.

b) The Hamiltonian introduced is finite on the space of Vassiliev invariants we introduced in the previous paper. This happens due to the detailed form in which the loop derivative acts on these states.

c) The Hamiltonian may be well defined on other spaces of states. For instance, on the space of functions of spin nets obtained by considering the Wilson nets along smooth holonomies (the original kind of function that was first considered as loop differentiable), it is straightforward that the Hamiltonian identically vanishes (essentially, since the loop derivative is smooth, the integrals that before gained contributions due to the distributional character of the loop derivative now vanish).

d) The last two points show that something surprising is happening here, in the sense that we did not construct the Hamiltonian in an ad-hoc way to obtain properties b) and c). That is, we introduced a discretization of the singlydensitized Hamiltonian and it naturally turned out to be well defined on the Vassiliev invariants and to vanish on holonomies of smooth connections. This might be pointing to a certain "naturalness" of the Vassiliev invariant space in the context of quantum gravity. In spite of the a priori huge ambiguity in the regularization of the singly-densitized 
Hamiltonian, it is difficult to imagine a regularization involving the loop derivative that would not be finite on the Vassiliev invariants. In addition, we have not introduced in our construction any ad-hoc "renormalization" of the operator to obtain finiteness (as one had to do in the doubly-densitized case).

We will now proceed to discuss the issue of the constraint algebra.

\section{CONSISTENCY OF THE CONSTRAINT ALGEBRA: PRELIMINARIES}

The operators we introduced are written purely in terms of the loop derivative and integrals along edges of the spin net. One can discuss the successive action of such operators without making specific reference to a space of states on which one is acting upon. Such a calculation would be "formal" in the sense that terms arising in it could fail to be well defined when acting on particular spaces of states. This is the underlying philosophy of the kind of (unregulated) formal calculations some of us undertook in reference [8]. Such an approach can be pursued with the regulated operators we introduce in this paper. The fact that the operators are regulated and that they might act on the space of diffeomorphism invariant states will imply certain departures from the specifics of the calculations of reference [8]. If one pursues calculations at this level of generality, one cannot expect to reproduce the classical Poisson algebra at the level of quantum commutators without making some assumptions about the distributional behavior of the various quantities involved in the calculations. One will recover terms that correspond to the classical ones but generically there will be additional terms. When one particularizes to a certain "habitat" of wavefunctions, these extra terms in many cases will vanish. However, it is not excluded that on certain habitats pathologies could appear. It would be an interesting exercise to pursue the calculation in general and then particularize to various "habitats". This, however, is a complex and lengthy calculation in terms of loop calculus. We will not attempt a calculation at this level of generality here. In this paper we will concentrate on computing the constraint algebra on a series of habitats on which we recover the classical constraint algebra at the level of quantum commutators. These habitats will include as particular cases spaces of diffeomorphism invariant functions on which the appropriate algebra will be recovered.

\section{A. Functions of spin nets with "marked vertices"}

A point to be addressed when studying the constraint algebra is that, given the action of the Hamiltonian constraint we have introduced, if one starts with a function of spin networks, when one acts with a Hamiltonian constraint one ends with a more general object. This can be explicitly seen for instance in equation (26). What we see is that the result of the action of a Hamiltonian on a function of spin networks is a function of spin networks times a "vertex function", that is, a function dependent on the position of the vertex where the Hamiltonian acts. If one wishes to compute the constraint algebra, the action of the second operator in the calculation of the algebra therefore takes place on such a space of functions. One can view these functions as simply more general functions of spin networks, since after all the information of the position of the vertices comes with a given spin network. This is possible, but one has to exhibit that dependence -of the vertices on the spin networks- in an explicit enough way that operators like the loop derivative have an appropriate action on such a dependence. One is initially tempted to say that the loop derivative simply ignores the extra factors, since they do not appear to contain an explicit loop dependence. But the position of the vertices depends on the edges of the spin network (more precisely, a vertex is defined by the intersection point of at least three edges of the net), and therefore the vertex function should be affected by the loop derivative operator. To operate with the loop derivative over this kind of functions, we need to make more explicit the implicit functional dependence $v[e(s)]$ of the vertex function. We limit the analysis to the case of trivalent vertices. Given three arbitrary open paths $\gamma_{i}(i=1,2,3)$ with a common origin $o$, we define the quantity,

$$
R_{c}(M, \epsilon, \gamma):=\frac{1}{2} \epsilon_{c d f} \int_{\overline{\gamma_{3}} \circ \gamma_{1}} d x_{1}^{d} \int_{\overline{\gamma_{3}} \circ \gamma_{2}} d x_{2}^{f}\left(M\left(x_{1}\right)+M\left(x_{2}\right)\right) \frac{\Theta_{\epsilon}\left(x_{1}-x_{2}\right)}{\epsilon^{2}},
$$

where $\gamma$ denotes generically the three curves $\gamma_{1}, \gamma_{2}, \gamma_{3}, \Theta_{\epsilon}(y)$ is 1 if $|y|<\epsilon$ and zero otherwise, and $M(x)$ is a scalar function defined on the manifold. It is assumed that the three paths are oriented and we denote by the overbar the path in the reversed orientation. We also assume that, starting at $o$, the three curves overlap in a finite segment and then they separate following disjoint paths, as it is shown in the figure. 


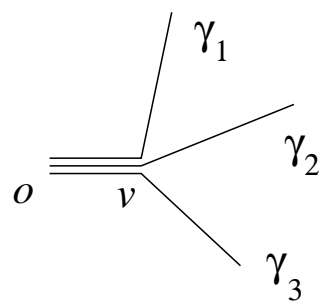

FIG. 1. The three open paths.

We call $v$ the bifurcation point of the three curves. It is immediate to show that,

$$
R_{c}(M, \epsilon, \gamma)=M(v) R_{c}(1, \epsilon, \gamma)+O(\epsilon),
$$

and,

$$
M_{\epsilon}(v):=\frac{\vec{R}(M, \epsilon, \gamma) \cdot \vec{R}(1, \epsilon, \gamma)}{|R(1, \epsilon, \gamma)|^{2}}=M(v)+O(\epsilon)
$$

The above expressions can be used to calculate the loop derivative of a vertex function. Given a vertex of the net with edges $e_{1}, e_{2}, e_{3}$, we identify the portion of the curve $\gamma_{i}$ going form $v$ to the end point with the portion of the edge $e_{i}$ from the vertex to some intermediate point. This automatically guarantees that the point $v$ defined through the family of curves $\gamma_{i}$ coincides with the chosen vertex of the graph. If the end points of the edges $e_{1}, e_{2}, e_{3}$ lie outside of the ball of radius $\epsilon$ centered at $v$, then one has the identity $R_{c}\left(M, \epsilon, \gamma_{1}, \gamma_{2}, \gamma_{3}\right)=R_{c}\left(M, \epsilon, e_{1}, e_{2}, e_{3}\right) \equiv R_{c}(M, \epsilon, e)$.

We can now proceed to compute explicitly the loop derivative. For any function $F(x)$ we have ( [9] chapter 1),

$$
\Delta_{a b}^{(e)}\left(\pi_{v}^{z}\right) \int_{e} d x^{c} F(x)=\delta_{[b}^{c} \partial_{a] z} F(z) .
$$

Using this result it is straightforward to calculate the loop derivative of $R_{c}(M, \epsilon, e)$,

$$
\begin{aligned}
\Delta_{a b}^{\left(e_{1}\right)}\left(\pi_{v}^{z}\right) R_{c}(M, \epsilon, e)= & \frac{\epsilon_{c d f}}{2 \epsilon^{2}} \int_{\overline{\bar{e}_{3} \circ e_{2}}} d y^{f}\left\{\delta_{[b}^{d} \partial_{a] z} M(z) \Theta_{\epsilon}(z-y)\right. \\
& \left.+(M(z)+M(y)) \delta(\epsilon-|z-y|) \frac{\delta_{[b}^{d}(y-z)_{a]}}{|z-y|}\right\} .
\end{aligned}
$$

The loop derivative of $M_{\epsilon}(v)$ is evaluated using the above result and the Leibniz' rule.

Now that we know how to compute the loop derivative of a vertex function, we can consider the construction of a "habitat" of functions of spin networks with marked points of the definite following kind:

$$
\psi_{\epsilon}(s, M, \Omega)=\sum_{v \in s} M_{\epsilon}(v) \Omega\left(\vec{J}_{v}\right) \psi(s) \equiv O_{\epsilon}(M, \Omega) \psi(s)
$$

where $\Omega\left(\vec{J}_{v}\right)$ is a group factor depending on the spins of the edges incident at $v$. This type of dependence on the vertex is precisely the one that appears in the action of the Hamiltonian constraint (24). In the last identity, we express the relationship between $\psi_{\epsilon}(s, M, \Omega)$ and $\psi(s)$ through an operator $O_{\epsilon}(M, \Omega)$. It is clear that, in the limit $\epsilon \rightarrow 0$, one gets as a result a function of the spin network times a function of the vertices and incoming spins,

$$
\psi(s, M, \Omega) \equiv O(M, \Omega) \psi(s):=\lim _{\epsilon \rightarrow 0} \psi_{\epsilon}(s, M, \Omega)=\sum_{v \in s} M(v) \Omega\left(\vec{J}_{v}\right) \psi(s) .
$$

To define the action of the constraints on these kinds of functions we will study the action of the constraints before taking the limit $\epsilon \rightarrow 0$ in these expressions in a suitable way. Let us start by considering the diffeomorphism constraint,

$$
\begin{aligned}
C(\vec{N}) \psi(s, M, \Omega) & =\lim _{\left\{\epsilon, \epsilon^{\prime}\right\} \rightarrow 0} C_{\epsilon^{\prime}}(\vec{N}) O_{\epsilon}(M, \Omega) \psi(s) \\
& =\lim _{\left\{\epsilon, \epsilon^{\prime}\right\} \rightarrow 0}\left[\left\{C_{\epsilon^{\prime}}(\vec{N}) O_{\epsilon}(M, \Omega)\right\} \psi(s)+O_{\epsilon}(M, \Omega) C_{\epsilon^{\prime}}(\vec{N}) \psi(s)\right] .
\end{aligned}
$$


In the second term the diffeomorphism acts on $\psi(s)$ leaving all the factors unaffected. This term is immediate to evaluate (and in the case of diffeomorphism invariant $\psi(s)$ 's vanishes), so we will not discuss it further. In order to analyze the first contribution it is convenient to make the following intermediate calculation,

$$
\begin{aligned}
C_{\epsilon^{\prime}}(\vec{N}) R_{c}(M, \epsilon, e)= & \sum_{e_{m}} \int d^{3} z \int_{e_{m}} d x^{b} \frac{N^{a}(x)+N^{a}(z)}{2} f_{\epsilon^{\prime}}(x, z) \Delta_{a b}^{(m)}\left(\pi_{x}^{z}\right) R_{c}(M, \epsilon, e) \\
= & \frac{\epsilon_{c d f}}{4 \epsilon^{2}} \int_{\overline{e_{3} \circ e_{2}}} d y^{f} \int_{e_{1}} d x^{b} \int d^{3} z\left(N^{a}(x)+N^{a}(z)\right) f_{\epsilon^{\prime}}(x, z)\left\{\delta_{[b}^{d} \partial_{a] z} M(z) \Theta_{\epsilon}(z-y)\right. \\
& \left.+(M(z)+M(y)) \delta(\epsilon-|z-y|) \frac{\delta_{[b}^{d}(y-z)_{a]}}{|z-y|}\right\}+ \text { cyclic permutations in } e_{1}, e_{2}, e_{3},
\end{aligned}
$$

where we have used (32). In the evaluation of this quantity, the result depends on the order in which the limits are taken. If one takes the limit $\epsilon \rightarrow 0$ first, one finds that the diffeomorphism annihilates $R_{c}$. This means that $\lim _{\epsilon \rightarrow 0} C_{\epsilon^{\prime}}(\vec{N}) M_{\epsilon}(v)=0$. On the other hand, if we take the limit $\epsilon^{\prime} \rightarrow 0$ first one gets the result,

$$
\lim _{\epsilon^{\prime} \rightarrow 0} C_{\epsilon^{\prime}}(\vec{N}) R_{c}(M, \epsilon, e)=\left[(\vec{N} \cdot \nabla M)(v)+M(v) \lim _{\epsilon^{\prime} \rightarrow 0} C_{\epsilon^{\prime}}(\vec{N})\right] R_{c}(1, \epsilon, e)+O(\epsilon),
$$

and,

$$
\lim _{\epsilon \rightarrow 0} \lim _{\epsilon^{\prime} \rightarrow 0} C_{\epsilon^{\prime}}(\vec{N}) M_{\epsilon}(v)=(\vec{N} \cdot \nabla M)(v)
$$

With this choice we see that the action of the diffeomorphism is what one would have expected geometrically: it Lie-drags the dependence of $M$ on the position of the vertex. This action immediately ensures that the action of the diffeomorphism constraint closes the appropriate commutator algebra on the space of functions we are considering, provided $\psi(s)$ is either invariant (like in the case of the Vassiliev invariants) or is such that the diffeomorphism constraint has the natural geometric action. In the general case,

$$
C(\vec{N}) O(M, \Omega) \psi(s)=O\left(N^{a} \partial_{a} M, \Omega\right) \psi(s)+O(M, \Omega) C(\vec{N}) \psi(s) .
$$

Based on these calculations for the diffeomorphism, let us now explore the action of the Hamiltonian constraint on the habitat we have been discussing. From (21) and (32) we get ( $\epsilon^{\prime}$ is now the scale parameter of the triangulation adapted to the spin network),

$$
\begin{aligned}
& H_{\epsilon^{\prime}}(N) R_{c}(M, \epsilon, e)=\frac{8}{3 G} e_{2}^{a} e_{3}^{b} \rho_{1}\left(\vec{J}_{v}\right) \epsilon_{c d f} \frac{\epsilon^{2}}{\epsilon^{\prime 2}} \int d^{3} z \int_{\bar{e}_{3} \circ e_{2}} d y^{f} f_{\epsilon^{\prime}}(z ; v) \times \\
& \left\{\delta_{[b}^{d} \partial_{a] z} M(z) \Theta_{\epsilon}(z-y)+(M(z)+M(y)) \delta(\epsilon-|z-y|) \frac{\delta_{[b}^{d}(y-z)_{a]}}{|z-y|}\right\}+\text { cyclic permut. in } e_{1}, e_{2}, e_{3},
\end{aligned}
$$

where we have written $f_{\epsilon^{\prime}}(y ; v) \equiv f_{\square_{v}}(y)$ in order to make explicit the dependence of the triangulation with $\epsilon^{\prime}$. Notice that,

$$
\lim _{\left\{\epsilon, \epsilon^{\prime}\right\} \rightarrow 0} \frac{\epsilon^{2}}{\epsilon^{\prime 2}} \int d^{3} z \int_{e} \Theta_{\epsilon}(z-y) f_{\epsilon^{\prime}}(z ; v)=0
$$

and,

$$
\lim _{\left\{\epsilon, \epsilon^{\prime}\right\} \rightarrow 0} \frac{\epsilon^{2}}{\epsilon^{\prime 2}} \int d^{3} z \int_{e} d y^{f} \delta(\epsilon-|z-y|) \frac{(y-z)_{a}}{|z-y|} f_{\epsilon^{\prime}}(z ; v)=0,
$$

irrespective of the order in which the limits are taken. This means that,

$$
\lim _{\left\{\epsilon, \epsilon^{\prime}\right\} \rightarrow 0} H_{\epsilon^{\prime}}(N) M_{\epsilon}(v)=0 .
$$

and taking into account (34),

$$
H(N) O(M, \Omega) \psi(s)=O(M, \Omega) H(N) \psi(s) .
$$

This is an important result. It will imply that the Hamiltonian constraints will commute with each other. We will discuss this in detail in the next section. 


\section{B. The right hand side of the commutator of two Hamiltonians}

In the classical theory, the Poisson bracket of two smeared Hamiltonian constraints is given by,

$$
\{H(N), H(M)\}=\int d^{3} x \omega_{a}(x) q^{a b}(x) \tilde{C}_{b}(x)
$$

with the one-form $\omega_{a}=\left(N \partial_{a} M-M \partial_{a} N\right)$ and $\tilde{C}_{b}(x)=\tilde{E}_{i}^{b}(x) F_{a b}^{i}(x)$ is the (unsmeared) diffeomorphism constraint. That is, the right hand side of this expression is a diffeomorphism generated by the vector obtained by contracting the one-form $\omega_{a}$ with a double-contravariant metric. If one wishes this expression to have a quantum counterpart, it implies realizing the product of the doubly contravariant metric times a diffeomorphism as a quantum operator. In order to do this, we need to re-express it in terms of variables that are suitable for the Ashtekar formulation. This is done via the following classical identities, as discussed by Thiemann [10,

$$
q^{a b}(x)=\frac{1}{4} \epsilon^{a c d} \epsilon_{i j k} \epsilon^{b e f} \epsilon_{i l m} \frac{e_{c}^{j} e_{d}^{k}}{\sqrt{\operatorname{det}(q)}} \frac{e_{e}^{l} e_{f}^{m}}{\sqrt{\operatorname{det}(q)}}
$$

and we recall the relationship of the covariant (undensitized) triads with the more usual contravariant densitized triads, $\tilde{E}_{i}^{a}=\epsilon_{i j k} \epsilon^{a b c} e_{b}^{j} e_{c}^{k}$. We can now use the key identity, due to Thiemann,

$$
2 G e_{a}^{i}(x)=\left\{A_{a}^{i}(x), V\right\}
$$

to obtain,

$$
q^{a b}(x)=\frac{1}{16 G^{2}} \epsilon^{a c d} \epsilon_{i j k} \epsilon^{b e f} \epsilon_{i l m} \frac{\left\{A_{c}^{j}(x), V\right\}\left\{A_{d}^{k}(x), V\right\}}{\sqrt{\operatorname{det}(q)(x)}} \frac{\left\{A_{e}^{l}(x), V\right\}\left\{A_{f}^{m}(x), V\right\}}{\sqrt{\operatorname{det}(q)(x)}}
$$

This expression cannot be easily promoted in a direct way to an operator due to the denominators involving the determinant of the metric. We can achieve this noting that the Poisson bracket of the connection and the volume depends only on local information of the volume operator in the surrounding of the point $x$. We can therefore replace in the above expression the volume of the whole manifold $V$, by a "localized" volume (as discussed by Thiemann [10]) $V\left(\mathcal{R}_{x}\right)$, given by,

$$
V\left(\mathcal{R}_{x}\right)=\int d^{3} y \Theta_{\mathcal{R}_{x}}(y) \sqrt{\operatorname{det} q(y)}
$$

where $\mathcal{R}_{x}$ is an infinitesimal closed region around the point $x$ and $\Theta_{\mathcal{R}_{x}}(y)$ is the characteristic function associated with this region. The following identity holds,

$$
\left\{A_{c}^{i}(x), V\right\}=\left\{A_{c}^{i}(x), V\left(\mathcal{R}_{x}\right)\right\}
$$

and in addition,

$$
\lim _{\mathcal{R} \rightarrow 0} \frac{V\left(\mathcal{R}_{x}\right)}{\mathcal{V}_{\mathcal{R}_{x}}}=\sqrt{\operatorname{det}(q)(x)}
$$

where $\mathcal{V}_{\mathcal{R}_{x}}$ is the Euclidean volume of the infinitesimal region $\mathcal{R}_{x}$ given by a fiducial flat metric (we will see that the final result does not depend on the fiducial metric). The above expressions allow us to absorb the denominators involving the determinant of the metric into Poisson brackets. Using this results we write (48) in the form,

$$
\begin{aligned}
q^{a b}(x)= & \frac{1}{G^{2}} \lim _{\mathcal{R} \rightarrow 0} \int d^{3} z \delta(z-x) \mathcal{V}_{\mathcal{R}_{z}} \times \\
& \epsilon^{a c d} \epsilon_{i j k} \epsilon^{b e f} \epsilon_{i l m}\left\{A_{c}^{j}(z), \sqrt{V\left(\mathcal{R}_{z}\right)}\right\}\left\{A_{d}^{k}(z), \sqrt{V\left(\mathcal{R}_{z}\right)}\right\}\left\{A_{e}^{l}(z), \sqrt{V\left(\mathcal{R}_{z}\right)}\right\}\left\{A_{f}^{m}(z), \sqrt{V\left(\mathcal{R}_{z}\right)}\right\}
\end{aligned}
$$

We now discretize the integral introducing the triangulation $\{\diamond\}=\left\{\square_{v}, \Delta^{\prime}\right\}$ of space defined in section II,

$$
\begin{aligned}
q^{a b}(x)= & \frac{1}{G^{2}} \lim _{\mathcal{R} \rightarrow 0} \lim _{\diamond \rightarrow 0} \sum_{\diamond} \mathcal{V}_{\mathcal{R}_{v}} \Theta_{\diamond}(x) \times \\
& \epsilon^{a c d} \epsilon_{i j k} \epsilon^{b e f} \epsilon_{i l m}\left\{A_{c}^{j}(v), \sqrt{V\left(\mathcal{R}_{v}\right)}\right\}\left\{A_{d}^{k}(v), \sqrt{V\left(\mathcal{R}_{v}\right)}\right\}\left\{A_{e}^{l}(v), \sqrt{V\left(\mathcal{R}_{v}\right)}\right\}\left\{A_{f}^{m}(v), \sqrt{V\left(\mathcal{R}_{v}\right)}\right\}
\end{aligned}
$$


where we have identified $v \equiv v_{\diamond}$ to simplify the notation. Using (8) we replace the $\epsilon^{a c d}$ and $\epsilon^{\text {def }}$ in terms of the volume of the elementary regions and the edges $u$ of the tetrahedra, and we join four of the $u$ 's with the $A$ 's to construct holonomies along the edges of the triangulation,

$$
q^{a b}(x)=\frac{1}{36 G^{2}} \lim _{\mathcal{R} \rightarrow 0} \lim _{\diamond \rightarrow 0} \sum_{\diamond} \alpha_{\diamond}^{2} \frac{\mathcal{V}_{\mathcal{R}_{v}}^{2}}{\mathcal{V}_{\diamond}^{2}} \Theta_{\diamond}(x) u_{p}^{a} u_{s}^{b} Q_{\diamond}^{p s}\left(\mathcal{R}_{v}\right)
$$

where we have defined,

$$
\begin{aligned}
Q_{\diamond}^{p s}\left(\mathcal{R}_{v}\right):= & \epsilon^{p q r} \epsilon^{s t n} \epsilon_{i j k} \epsilon_{i l m} \operatorname{Tr}\left[\tau^{j} h\left(u_{q}\right)\left\{h^{-1}\left(u_{q}\right), \sqrt{V\left(\mathcal{R}_{v}\right)}\right\}\right] \operatorname{Tr}\left[\tau^{k} h\left(u_{r}\right)\left\{h^{-1}\left(u_{r}\right), \sqrt{V\left(\mathcal{R}_{v}\right)}\right\}\right] \times \\
& \operatorname{Tr}\left[\tau^{l} h\left(u_{t}\right)\left\{h^{-1}\left(u_{t}\right), \sqrt{V\left(\mathcal{R}_{v}\right)}\right\}\right] \operatorname{Tr}\left[\tau^{m} h\left(u_{n}\right)\left\{h^{-1}\left(u_{n}\right), \sqrt{V\left(\mathcal{R}_{v}\right)}\right\}\right] .
\end{aligned}
$$

We are now ready to promote the above expression as an operator acting over the spin network wavefunctions, which we assume to be the loop transform of a state in the connection representation. As in the case of the Hamiltonian constraint, we adapt the triangulation to the graph of the spin network choosing the points $\{v\}$ of the boxes $\left\{\square_{v}\right\}$ coincident with the vertices of the spin network, and we choose the regions $\mathcal{R}_{v}=\square_{v}^{\prime}$ equal to that defined by the triangulation around $v$ but with a length scale $\epsilon^{\prime}$. With this prescriptions we get from (54),

$$
q^{a b}(x) \psi(s)=\frac{16}{9 G^{2}} \lim _{\left\{\epsilon, \epsilon^{\prime}\right\} \rightarrow 0} \sum_{v \in s} \frac{\epsilon^{\prime 6}}{\epsilon^{6}} \Theta_{\epsilon}(x ; v) \int_{e_{p}} d w^{a} \Theta_{\epsilon}(y ; w) \int_{e_{s}} d t^{b} \Theta_{\epsilon}(t ; v) Q^{p s}(v) \psi(s),
$$

where we have used (11) and $\Theta_{\epsilon}(x ; v) \equiv \Theta_{\square_{v}}(x)$ with $\epsilon$ the length scale of the regions $\square_{v}$. The action of the operator $Q^{p s}(v)$ on the spin network wavefunctions is calculated as usual through its action on the Wilson net appearing in the loop transform. It is important to notice that this operator looses all dependence with the scale parameters involved in the triangulation and the localized volume. This is due to the fact that, acting on the Wilson net, the holonomies $h(u)$ that appear in the quantum version of (55) generate (in the limit $\diamond \rightarrow 0$ ) finite recoupling coefficients and that the action of the volume operator is local (it depends only on the vertex included in the region $\mathcal{R}_{v}$ ).

With this result at hand it is straightforward to quantize the right hand side of the Poisson bracket of two smeared Hamiltonian constraints. From the classical expression we immediately write,

$$
\begin{aligned}
\widehat{R H S} \psi(s) & =\int d^{3} x \omega_{a}(x) q^{a b}(x) C_{b}(x) \\
& =\lim _{\delta \rightarrow 0} \sum_{e \in s} \int d^{3} x \int_{e} d y^{c}\left[\omega_{a}(x) q^{a b}(x)+\omega_{a}(y) q^{a b}(y)\right] f_{\delta}(x, y) \Delta_{c b}^{(e)}\left(\pi_{y}^{x}\right) \psi(s),
\end{aligned}
$$

where we have used the regularized expression of the unsmeared diffeomorphism operator (see the companion paper, section II.B). Introducing now the regularized metric operator we get,

$$
\begin{aligned}
\widehat{R H S} \psi(s)= & \frac{16}{9 G^{2}} \lim _{\left\{\epsilon, \epsilon^{\prime}, \delta\right\} \rightarrow 0} \sum_{e \in s} \sum_{v \in s} \frac{\epsilon^{\prime 6}}{\epsilon^{6}} \int d^{3} x \int_{e} d y^{c}\left[\omega_{a}(x) \Theta_{\epsilon}(x ; v)+\omega_{a}(y) \Theta_{\epsilon}(y ; v)\right] f_{\delta}(x, y) \times \\
& \int_{e_{p}} d w^{a} \Theta_{\epsilon}(w ; v) \int_{e_{s}} d t^{b} \Theta_{\epsilon}(t ; v) Q^{p s}(v) \Delta_{c b}^{(e)}\left(\pi_{y}^{x}\right) \psi(s) .
\end{aligned}
$$

At this point it is worthwhile mentioning that this expression has a regularization ambiguity, given by the two regularization parameters $\epsilon$ and $\epsilon^{\prime}$. The latter was introduced, as we discussed above, in the definition of the localized volume operator. This ambiguity, associated with the fact that the localized volume and the ordinary one are the same on these spaces of functions was first noticed by Lewandowski and discussed in [11]. If one chooses $\epsilon=\epsilon^{\prime}$ one notices quickly that the expression for the right-hand-side vanishes. The powers $\frac{\epsilon^{\prime 6}}{\epsilon^{6}}$ cancel and one is left with two integrals along $w, t$ which are of order $\epsilon$ each. The rest of the expression is simply the action of a diffeomorphism. Assuming that the latter is finite, the expression therefore vanishes.

If we trace back the origin of this cancellation, one notices that the expression (56) for the contravariant metric operator vanishes identically over spin network states of any kind, in particular, the Vassiliev invariants we are considering in this paper. This property is a consequence of the following two peculiarities of the quantization of $q^{a b}$ in terms of spin network wavefunctions: the sum over the triangulation reduces to a sum over the vertices of the spin net (which includes a finite number of terms), and in each term the result of $Q^{p s}(v) \psi(s)$ is finite. Notice that in the classical result (48) one has an infinite sum of $Q_{\diamond}^{p s}\left(\mathcal{R}_{v}\right)$, each of which tends to zero in the limit $\{\diamond, \mathcal{R}\} \rightarrow 0$. 
This limit gives in general a nonzero result. But in (56) we have instead a finite sum of terms $Q^{p s}(v) \psi(s)$, which are independent of $\epsilon$ and $\epsilon^{\prime}$. This fact alters the power counting of the factors $\epsilon$ and $\epsilon^{\prime}$ in such a way that all the terms tends naturally (i.e. choosing $\epsilon=\epsilon^{\prime}$ ) to zero as the triangulation shrinks to a point.

That the right-hand-side may vanish was already observed in [11]. Could one "tune" the limits in $\epsilon$ and $\epsilon^{\prime}$ so this quantity is nonvanishing? One could, but the result would be dependent on the background structures introduced to regularize. If one made the expression non-vanishing it would be proportional to the normalized tangent vectors at the intersection, which are background dependent. This is not surprising: there is no naturally defined second order symmetric contravariant tensor defined in a manifold without metric. This result is quite strong. It implies that the commutator of two Hamiltonians will have to vanish if one wishes to have consistency. We anticipated that this would happen in the previous subsection and we will now see in detail how it happens.

As a final remark, we notice that if one wished to define the doubly covariant metric, it is straightforward to compute it using the identities introduced by Thiemann and one finds that regularized with the same procedures we followed up to now, it diverges. Should one worry about a theory of quantum gravity where the doubly contravariant metric vanishes and the doubly covariant metric diverges? We will return to this in the discussion section.

\section{CONSISTENCY OF THE CONSTRAINT ALGEBRA: HABITATS}

\section{A. Habitat I: holonomies of smooth connections}

The loop derivative was originally introduced in the context of Yang-Mills theories, where the natural functions to act upon were holonomies of smooth connections. These functions are not the most natural ones to consider in the context of diffeomorphism invariant theories like general relativity, but our constraints are well defined on them, so we can consider them to be a "habitat" where to test the constraint algebra, at least as a mathematical exercise. On these kinds of functions the diffeomorphism constraints that we have introduced here are known to close the appropriate algebra in the limit in which regulators are removed (see [8], although the regularization is slightly different than the one we use in this paper, it is immediate to check that the same calculations go through). We therefore will not repeat the calculation here. The Hamiltonian constraint vanishes identically on this habitat. Starting from equation (21), if the loop derivative is a smooth function, one is left with two one-dimensional integrals along the edges of a cell of a finite function. In the limit in which the triangulation is shrank, the result vanishes. The commutator of a diffeomorphism with a Hamiltonian therefore immediately reproduces the classical result.

The right-hand side of the commutator of two Hamiltonians that we introduced in the previous subsection also vanishes, here one has three one dimensional integrals along the edges of a triangulation of a quantity that goes as $\epsilon^{\prime 6} / \epsilon^{6}$. The final result therefore goes as $\epsilon^{\prime 6} / \epsilon^{3}$. Therefore, as long as one chooses $\epsilon^{\prime}$ shrinking to zero faster than $\sqrt{\epsilon}$, the right hand side vanishes.

On this space therefore, we reproduce the classical Poisson algebra at the level of the commutators. The diffeomorphisms close among themselves and the Hamiltonian vanishes identically. Could one simply claim that this is the "right" habitat to do quantum gravity? The answer is that this is unlikely. Although all states are solutions of the Hamiltonian constraint, this space does not contain any solution to the diffeomorphism constraint. Solutions to the diffeomorphism constraint in terms of holonomies can only be constructed as infinite superpositions, functional integrals or "group averaging", and in these cases one includes connections that are not smooth.

An interesting point is that in this habitat, since one does not have solutions of the diffeomorphism constraint but has solutions to the Hamiltonian constraint, the only way that one could achieve consistency in the algebra is if the right hand side of the commutator of two Hamiltonians vanishes, which is the case.

Another point to consider is that if one examines the expression of the commutator of two Hamiltonians, although both members vanish in the limit in which one shrinks the triangulation — given the smoothness of the loop derivativeaway from the limit the calculation is problematic. For instance, it is not clear what is the action of the volume operator on a state given by the loop derivative of a smooth holonomy. One might consider introducing a definition of the volume on this kind of space, but this has yet to be done in detail.

\section{B. Habitat II: The framing-independent Vassiliev invariants}

Remarkably, this space of invariants also leads to a reproduction of the classical Poisson algebra at a trivial level only (as usual, we limit our discussion to trivalent intersections). This is based on the fact proved in the appendix of the previous paper, that these invariants are annihilated by the loop derivative that appears in both the diffeomorphism and the Hamiltonian constraints and of the expression of the RHS. Therefore the ambient isotopic 
(framing-independent) Vassiliev invariants for spin networks with trivalent intersections are annihilated by all the constraints of quantum gravity and consistently, by the right hand side of the commutator of two Hamiltonian constraints. It should be pointed out that the space is in no way trivial: as we discussed in the appendix of the previous paper, the annihilation is a detailed property of the space of Vassiliev invariants, related to the decomposition of the invariants in framing independent and framing dependent components and the detailed structure of chord diagrams appearing in the coefficients. Hints that the Vassiliev invariants for trivalent intersections were annihilated by the Hamiltonian constraint were found for the lower invariants in terms of loops [12], [13], in the lattice [14].

One could mention as a more trivial example of a space with similar property the states based on diffeomorphism invariants of spin networks with no vertices, which are also trivially annihilated by all the constraints we consider. This is an extension of results also first suggested in the context of loops [15].

It should be remarked that this property of the loop derivative is not true for higher valence intersections. Future extensions of the Hamiltonian constraint to higher valence intersections could be tested in this habitat for non-trivial consistency.

\section{Habitat III: Vassiliev invariants}

By this space we mean the invariants that appear in the power series expansion of the expectation value of the Wilson net in a Chern-Simons theory in terms of the inverse coupling constant $\kappa$. In particular, the whole series is an example of such an invariant. Each coefficient of the series and certain portions of them are also examples. In general, these invariants are sums and products of the independent invariants that appear at each order, both framing-independent and framing-dependent (see previous paper). These states have the important property that the loop derivative that appears in the expressions of the constraints (which is evaluated on a path $\pi$ of infinitesimal length) can be rewritten simply as,

$$
\Delta_{a b}\left(\pi_{x}^{y}\right) V(s)=\sum_{e_{x} \in s} \int_{e_{x}} d z^{c} \epsilon_{a b c} \delta^{3}(z-y) V^{\prime}(s)
$$

where $e_{x}$ is the edge of the spin net containing the point $x$ and where the invariant $V^{\prime}(s)$ is another Vassiliev invariant of one order less than $V(s)$. What happens is that because of the infinitesimal length of $\pi$ one can rearrange the action of the loop derivative in terms of the original spin network $s$ using recoupling identities, at the expense of some additional group factors, which we reabsorb notationally in $V^{\prime}(s)$. We will see that we do not need the details of the relation of $V(s)$ to $V^{\prime}(s)$ for proving the consistency. As usual, all our discussion is limited to trivalent intersections. The relation above implies that for the Vassiliev invariants, one can write,

$$
H(N) V_{n}(s)=-\frac{1}{3 G} \sum_{v \in s} N(v) \nu_{\vec{J}_{v}} V_{n-1}(s) .
$$

This can be seen by considering equation 26) and recalling that $E(s, \kappa)=\sum_{n=0}^{\infty} V_{n}(s) \kappa^{n}$. In particular, we see that the action of the Hamiltonian on these states can be written (up to a constant factor which we will omit for simplicity) as,

$$
H(N) V_{n}(s)=O(N, \nu) V_{n-1}(s)
$$

\section{Commutator of two diffeomorphisms}

On this space, the diffeomorphism constraint vanishes identically, as we discussed in the previous paper. So the algebra of diffeomorphisms is trivially satisfied. Nontrivial commutators to be realized will be those of a diffeomorphism with a Hamiltonian and that of two Hamiltonians.

\section{Commutator of a diffeomorphism with the Hamiltonian}

In the case of a diffeomorphism with a Hamiltonian, since the wavefunctions are diffeomorphism invariant, of the

two terms of the commutator one is left with the one in which the diffeomorphism acts at the left. Since the action of the Hamiltonian on $V(s)$ is not diffeomorphism invariant, one should recover the proper action of the diffeomorphism 
through such term. Here the derivations of section III A become useful. We start from the expression (61), and then use (39) to get,

$$
C(\vec{N}) H(M) V_{n}(s)=O\left(N^{a} \partial_{a} M, \nu\right) V_{n-1}(s)+O(M, \nu) C(\vec{N}) V_{n-1}(s)=H\left(N^{a} \partial_{a} M\right) V_{n}(s) .
$$

and from here the correct commutator follows immediately. This implies that the quantum Hamiltonian transforms covariantly in a correct way. This calculation is one of the main differences of our construction with respect to the one of Thiemann [4], since in that context one does not consider an infinitesimal generator of diffeomorphisms.

\section{Commutator of two Hamiltonians}

As we discussed in section IIIA, the action of a Hamiltonian on a function with marked points as one gets after acting with a Hamiltonian on a Vassiliev invariant was given by expression (44), which we can combine with the action of the Hamiltonian (61) to get,

$$
H(N) H(M) V_{n}(s)=H(N) O(M, \nu) V_{n-1}(s)=O(M, \nu) H(N) V_{n-1}(s)=O(M, \nu) O(N, \nu) V_{n-2}(s) .
$$

And if one performs the calculation in the reverse order, one obtains the $O$ operators in the reverse order. However, these operators are multiplicative, so they commute. Therefore one has the result,

$$
[H(N), H(M)] V_{n}(s)=0
$$

which is the expected commutation relation on states that are invariant under diffeomorphisms.

\section{Habitat IV: diffeomorphism dependent functions}

The states we have already considered, where one has a Vassiliev invariant times a scalar function that depends on the position of the vertices of the spin network, are an attractive habitat where the diffeomorphism constraint does not vanish.

\section{Commutator of diffeomorphisms}

As discussed in section ( $\overline{\text { III A }}$ ) the diffeomorphisms on these states reduce to Lie dragging of the scalar functions of the vertices (see formula (39)). Since they correspond to a natural geometric action, the consistency of the algebra of diffeomorphisms is immediate.

\section{Commutator of diffeomorphism with a Hamiltonian}

We start from the action of a Hamiltonian on these kinds of states,

$$
H(N) O(M, \Omega) V_{n}(s)=O(M, \Omega) O(N, \nu) V_{n-1}(s)
$$

where we have used the fact that $O$ and $H$ commute and that the action of the Hamiltonian on these states produces a Vassiliev invariant of order lowered by one unit times a vertex function. We now act with a diffeomorphism,

$$
C(\vec{L}) H(N) O(M, \Omega) V_{n}=C(\vec{L}) O(M, \Omega) O(N, \nu) V_{n-1}(s),
$$

and now use the fact that Leibniz' rule applies to the action of the diffeomorphism constraint (stemming from the fact that it also applies to the action of the loop derivative),

$$
C(\vec{L}) O(M, \Omega) O(N, \nu) V_{n-1}(s)=O\left(L^{a} \partial_{a} M, \Omega\right) O(N, \nu) V_{n-1}+O(M, \Omega) O\left(L^{a} \partial_{a} N, \nu\right) V_{n-1},
$$

and we now reconstruct the Hamiltonian in the first and second terms, 


$$
\begin{aligned}
O\left(L^{a} \partial_{a} M, \Omega\right) O(N, \nu) V_{n-1}+O(M, \Omega) O\left(L^{a} \partial_{a} N, \nu\right) V_{n-1}= & O\left(L^{a} \partial_{a} M, \Omega\right) H(N) V_{n} \\
& +O(M, \Omega) H\left(L^{a} \partial_{a} N\right) V_{n},
\end{aligned}
$$

and using the fact that $H$ and $O$ commute, we get,

$$
C(\vec{L}) H(N) O(M, \Omega) V_{n}=H(N) O\left(L^{a} \partial_{a} M, \Omega\right) V_{n}+H\left(L^{a} \partial_{a} N\right) O(M, \Omega) V_{n}
$$

which we can rewrite as,

$$
C(\vec{L}) H(N) O(M, \Omega) V_{n}=H(N) C(\vec{L}) O(M, \Omega) V_{n}+H\left(L^{a} \partial_{a} N\right) O(M, \Omega) V_{n},
$$

from where we get the correct commutation relation,

$$
[C(\vec{L}), H(N)] O(M, \Omega) V_{n}=H\left(L^{a} \partial_{a} N\right) O(M, \Omega) V_{n}
$$

\section{Commutator of two Hamiltonians}

This calculation proceeds along the same lines as the one in the previous subsection, we essentially rewrite the action of the Hamiltonian in terms of the $O$ operator, and note that the $O$ operators commute,

$$
H(L) H(N) O(M, \Omega) V_{n}(s)=O(L, \nu) O(N, \nu) O(M, \Omega) V_{n-2}(s)=H(N) H(L) O(M, \Omega) V_{n}(s),
$$

and we therefore get that $[H(N), H(M)]=0$, which as we discussed before, is consistent with the representation in these spaces of functions of the right hand side of the quantum commutator.

\section{DISCUSSION}

\section{A. Summary}

We have presented a canonical quantization of the constraints of canonical general relativity. We represented the diffeomorphism and Hamiltonian constraints using two novel ingredients: the loop derivative to represent the field tensor $F_{a b}$ and the use of spaces related to the generalization of the Vassiliev invariants to spin networks (restricted to trivalent intersections) as wavefunctions. In terms of the latter we constructed several "habitats", including spaces of functions that are not invariant under diffeomorphisms and we checked that one obtained a consistent algebra of quantum commutators of the constraints. Consistency in this sense implies that the quantization of the canonical Poisson identities between the classical constraints is implemented correctly in the quantization. We have observed that this consistency is achieved at the price of having a vanishing right hand-side for the commutator of two Hamiltonians and that we can trace back this fact to the vanishing nature of the doubly covariant metric operator in these spaces of functions.

\section{B. Comparison with Thiemann's results}

We should point out common elements and differences with the quantization presented by Thiemann [4]. In Thiemann's case the Hamiltonian was implemented on the space of diffeomorphism invariant cylindrical functions of spin networks. On these spaces one does not have a well defined notion of the field tensor $F_{a b}$ and the functions are not loop-differentiable. In our case, the availability of the loop derivatives allows to have at hand a "differential calculus" that allows for several novel constructions. Examples of them are the calculations performed in computing the constraint algebra, and the possibility of finding novel solutions to the Hamiltonian constraint, based on the behavior of the Vassiliev invariants under loop differentiation.

Another difference involves the implementation of an infinitesimal generator of diffeomorphisms. In Thiemann's original construction one worked directly in terms of diffeomorphism invariant states and therefore one did not have an infinitesimal generator. The construction was extended by Lewandowski and Marolf [16] to "habitats" that are dependent on diffeomorphisms. With that extension, Thiemann's work achieves the same level of consistency that we have in this paper, in the sense that one can check non-trivial commutators of diffeomorphisms and diffeomorphisms 
with Hamiltonians. One still has the feature of a vanishing right hand side of the commutator of two Hamiltonians [11]. As we discussed in this paper, this feature appears as inescapable in the context of wavefunctions only dependent on spin networks as the ones we considered here, since as we mentioned one does not have enough structures to construct a naturally defined symmetric metric tensor.

Other differences arise insofar as the space of wavefunctions considered. In the case of Thiemann, one had spaces of functions with well defined inner products, which allow to discuss normalizability and study the spaces of solutions with a level of rigor that is not available currently in our approach, since we do not have an inner product on the habitats we are considering. It might not be impossible to find a suitable inner product with the same techniques that led to the construction of the measures on the spaces of cylindrical functions, but it has not been achieved yet. Concerning solutions of the constraints, one has some available both in our approach and in Thiemann's, that appear as quite distinct in their features. In Thiemann's approach, the solutions are obtained using group averaging techniques. This leads to structures like "tassels" and others [17] in which accumulation of lines and vertices take place. In our approach, one has a number of solutions of the Hamiltonian constraint (the framing independent Vassiliev invariants for trivalent intersections) that do not depend on such structures. On the other hand, they appear as "trivial" solutions in the sense that they may not exist if one considers intersections of higher valences. In our approach one may also construct solutions to the Hamiltonian constraint with a cosmological constant, following similar ideas that led to the construction of states in terms of loops 18. Although we have not pursued this in detail yet, it appears quite plausible that these types of solutions exist, given the structure of the extra term due to the cosmological constant in the Hamiltonian constraint in terms of the spin network approach [19].

Thiemann's approach has also been studied in $2+1$ dimensions [21], and appears to lead to a satisfactory quantization, provided one chooses in an ad-hoc way an inner product that rules out certain infinite dimensional set of solutions. In a forthcoming paper we will discuss the quantization of $2+1$ dimensional gravity using an approach that has elements in common with the one we pursue here, in particular the requirement of loop differentiability of the states. We will see that this requirement limits us (at least for low valence intersections) to the correct solution space in a natural way.

\section{Is the theory satisfactory?}

The reader might find unsatisfactory that the right hand side of the commutator of two Hamiltonians vanishes. Even more unsatisfactory may appear the fact that this is due to the vanishing of the doubly covariant metric in this approach to quantum gravity. In this subsection we will address this and other issues.

The first question that one may raise is if this is not just a pathology that is introduced by our limitation to trivalent intersections for reasons of calculational convenience. After all, one knows that this subspace in in some sense degenerate since the volume operator vanishes identically. Unfortunately, it appears unlikely that extending our results to higher valent intersections (to which we do not see any technical obstruction, apart from greater calculational complexity) will change things insofar as the commutator of two Hamiltonians. The action of the Hamiltonian constraint on higher valent intersections is more complicated largely because the volume operator and the action of the loop derivative cannot be reduced to a simple group-dependent prefactor times the original state, but will in general involve a linear combination of states, with a non-trivial group-dependent matrix of coefficients. However, the dependence of the Hamiltonian on the smearing function will remain as a multiplicative one. Therefore it appears that the commutator of two Hamiltonians will again vanish. Moreover, the reasons we gave for the vanishing of the right hand side of the commutator are independent of the valences of the intersections of the spin networks. The expression for the doubly-covariant metric will be more complicated, but will still include a prefactor with the same dependence on the regulators as in the trivalent case and it will vanish. One therefore expects consistency at the same level we achieved for the trivalent case.

Can one consider satisfactory a theory with a vanishing doubly contravariant metric (and a divergent doubly covariant metric)? The answer to this will only appear in a definitive way when one constructs physical predictions from the theory, which at the moment are lacking. One can get partial indications that the pathology might not be as severe as it appears at first sight from the fact that one can define reasonable quantities like areas, volumes and lengths in this framework in spite of having the ill defined metric operators. An attractive feature of the Vassiliev states is that other diffeomorphism invariant operators can be defined as well. For instance, if one considers the integral $\int \sqrt{g} \operatorname{Tr}\left(F_{a b} F^{a b}\right)$, given that the Chern-Simons states are such that the densitized triads are proportional to the magnetic vector constructed from the curvature, one finds that quantum representation of this integral is identical to the volume operator.

An important observation concerning how satisfatory is a theory with a vanishing metric operator like the one we propose is that in 2+1-dimensional gravity similar pathologies appear, yet the correct physical theory is recovered. 
If one pursues a quantization similar to Thiemann's f in $2+1=$ dimensions (this is discussed in detail in [21]) one notices that the Hamiltonians also commute. Moreover, the geometrical arguments leading to the vanishing of the $q^{a b}$ operator still hold in this context: there is no quantity one can build out of loop states that will yield a doubly contravariant symmetric tensor. Yet, it was shown in [21] that one can recover the correct physical theory. How can these apparently contradictory elements be reconciled? It has to do with the nature of the kinematical calculations we are performing. To check the "off shell" contraint algrebra one is required to operate with a kinematial space of wavefunctions that are not annihilated by the constraints. When one recovers "physics" one should really do it with states that are annihilated by the constraint. This can be carried out in detail in the $2+1$ dimensional case. Suppose one wishes to ask questions about the "metric" of the theory. The first observation is that on physical states one cannot define a metric tensor since the latter states are diffeomorphism invariant and the metric tensor is not. One could gauge fix a metric tensor, say by asking questions about the value of its components in a fixed coordinate system. These questions can be answered: the components of the metric in a fixed coordinate system can be related to the values of the invariant operators $T^{0}$ and $T^{1}$ in such a coordinate system (and therefore in general, since the latter are coordinate invariant). One therefore has well defined operators associated with the metric that can be evaluated in the physical space of states. But such operators have little to do with the $q^{a b}$ we introduced in the kinematical space. It is not obvious at all that the two operators will be related in any way since the former are non-vanishing whereas the latter vanishes identically.

There is a certain disquieting element in the last observation, since it seems to imply that one should be careful before drawing conclusions from calculations at a kinematical level. This in particular, implies all calculations involving the constraint algebra. When we set out to work on the current paper, our expectation was that reproducing the contraint algebra would be a strong test for our quantization procedure, that perhaps would rule out all but a few of the possible theories. What we have learned is that this is probably not the correct view on the issue: one can obtain consistency in many ways at a kinematical level (in particular with $q^{a b}=0$ ). One can construct many consistent theories of quantum gravity. And still, what the $2+1$ dimensional example shows is that the "physics" of all these theories is really deeply buried in the states that solve the constraints. In a sense this is good, since the theory we are proposing here differs significantly at this level from the one proposed by Thiemann and therefore further adds to our options for trying to match experimental results.

The observation that kinematical calculations may have quite nontrivial connections with "real physics" poses problems for computations that try to obtain heuristic physical insights from looking at properties of the kinematical states (the "weave" approach to the semiclassical theory). An example of this are our own results on gamma-ray-burst light dispersion [20]. From the $2+1$ example we see that quantum fluctuations in the "physical metric" (the ones one would expect to influence the propagation of matter fields) may not have a direct connection with fluctuations of the kinematical metric. Yet it is the latter that are used in the concrete computations of [20].

Concerning the latter point, it is worthwhile mentioning explicitly that the theory presented in this paper can be coupled to matter and have well defined expressions for the Hamiltonians of matter fields, which also involve the metric in a non-trivial way (the coupling of the theory to matter can, for instance, be achieved using the same construction as Thiemann [10]; or one could consider alternative settings in which the loop derivative is also used in the representation of certain matter fields). It is somewhat unfortunate that the remarkable results on black hole entropy that have been achieved within this context [23] do not involve in a detailed way the action of the Hamiltonian constraint in the "bulk" of the spacetime to be used as guideline for constructing the constraints, and therefore cannot distinguish at the moment our proposal from Thiemann's.

The vanishing of the doubly contravariant metric tensor, apart from appearing as a "robust" feature based only on elementary notions of covariance of the elements involved in constructing the spin network states, is the way in which our approach bypasses the "hermiticity problem" of the canonical quantization. As we mentioned, if one were to demand that the diffeomorphism and Hamiltonian constraints and the doubly covariant metric be Hermitian operators (since they correspond to real classical quantities), the commutator of two Hamiltonians (which is Hermitian) cannot be simply equal to the product of a metric times a diffeomorphism (which is not Hermitian). One could fix the Hermiticity of the right hand side by "symmetrizing" the operator, but then one could face an anomaly problem, since the diffeomorphism constraint would not act to the right of the product. This difficulty is bypassed (in the context of non-diffeomorphism invariant states) if the metric vanishes. It should be noticed that it is not obvious that one should promote the constraints to Hermitian operators, for instance, see [22] for counterexamples in the context

\footnotetext{
${ }^{4}$ In a forthcoming paper we will present a quantization of $2+1$ dimensional gravity along the same lines as the one proposed in this paper, and the observations we make in this subsection apply to it as well. We will also expand the discussion on the physical observables and their connection with the kinematical metric we present in this subsection.
} 


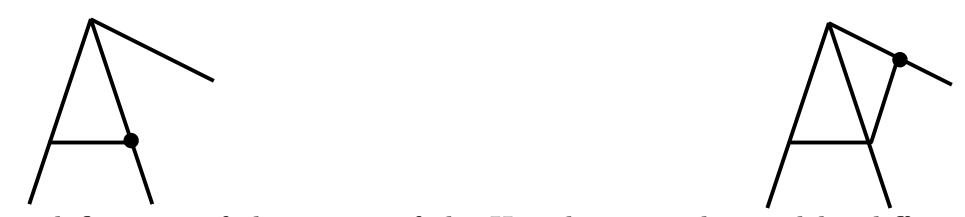

FIG. 2. Possible alternative definitions of the action of the Hamiltonian, obtained by different choices of path in the loop derivative.

of the quantization of non-unimodular gauge groups.

\section{Is the theory unique?}

Related with the issue of the lack of guidelines to construct the theory is the problem of uniqueness. We already have a manifest non-uniqueness in that the theory presented here and the theory introduced by Thiemann appear as both satisfactory yet distinct. The ambiguity is worse than this. Even if one stays within one general approach, for instance considering the space of Vassiliev invariants as "arena" for quantization, there are many ways in which one could implement the Hamiltonian constraint. We have not analyzed them in any detail, but some salient features of them are worthwhile mentioning. The ambiguities arise in the various limits involved in constructing the Hamiltonian. One of them is associated with the specific action of the loop derivative. The loop derivative is dependent on a path. In this paper we have taken such a path to coincide with one of the lines of the spin network. We then acted with the loop derivative and collapsed its action using recoupling identities. The final action of the Hamiltonian was therefore "ultra-local" in the sense that it returned a wavefunction with the same vertex structure times a vertex dependent prefactor (for valences higher than three one gets a linear combination with different spin weights for the intertwiners yet the topology of the vertex is unchanged). This may raise concerns that "super-selections" could appear in the sense that it could be easy to construct operators that commute with the Hamiltonian. One could define a different Hamiltonian in a straightforward way, by assuming a different topology for the path associated with the loop derivative. For instance, one could assume that the path starts at the vertex, advances along one of the edges of the spin network and then crosses towards another edge of the spin network along one of the edges of the tetrahedra introduced in the discretization. At the end of the path, the loop derivative acts. One then is left with an action of the Hamiltonian resembling the one introduced by Thiemann: the constraint produces a vertex dependent prefactor, but it also alters the structure of the spin network by "adding a line" at the vertex. A different proposal would be to have a path that starts at the vertex, advances along one of the edges of the spin network, crosses towards another edge along the tetrahedron and then continues to cross towards another edge of the spin network, as shown in figure 2 .

In the first of these proposals (as in Thiemann's), the Hamiltonian adds vertices called "exceptional" in the sense that they are planar vertices. The Hamiltonian vanishes identically on such vertices, because the volume vanishes on planar vertices, even if they are four-valent. In the second proposal, the intermediate vertex that is created is non-planar. This might raise hopes that the commutator of two Hamiltonians may be less trivial, since the second Hamiltonian acts at the newly created vertex, but more careful analyses seem to indicate that the Hamiltonians still commute [1]. If it they were not to commute one would still be faced with how to reproduce a similar vertex structure with the right hand side operator in a natural way. Summarizing this portion of the discussion: it appears that there is a non-trivial, possibly infinite, amount of ambiguity in the definition of the theory, that is not significantly constrained by the imposition of the correct quantum commutator algebra.

Related to the latter point is the fact that due to the vanishing of the metric, the consistency check provided by the commutator of two Hamiltonians is less detailed than if one had to prove the equality of non-vanishing operators. We already see that we have two distinct and apparently consistent, quantizations of the constraints (with several possible variants of each). It will require further study to determine if one quantization is "better" in the sense of reproducing expected results than the other. One possibility would be to consider the commutation of the Hamiltonian with various operators, and study if inconsistencies appear. This procedure, however, might be limited by the fact that most commutators have non-trivial right hand sides which will inevitably involve quite a bit of ambiguity at the time of their quantization.

Another point that might be raised is that these approaches appear confined to four dimensions and to the EinsteinHilbert action, and therefore one may have little hopes of making contact with other approaches, as those based on string theories. These approaches not only may present avenues to understand quantum gravity but they also have the attractive feature of unifying gravity with other interactions, a goal some may consider desirable in itself. It should be pointed out however, that progress is being made [24 towards describing $N$ dimensional general relativity 
in terms of connections. Although constructions like the ones we discussed here have not been pursued in detail in this context, they appear as plausible.

\section{E. Final remarks}

To conclude, we have at the moment canonical quantizations of general relativity (possibly coupled to matter) that appear as mathematically consistent at the kinematical level at which they have been studied. This level of consistency had never been achieved before in other approaches. Further exploration of the consequences of the quantization will be needed to determine if any of them are physically satisfactory theories of the quantum gravitational field. In particular the exploration of the space of states that solve the constraints and how physical quantities can be evaluated on them appears as a natural next step in the quantization program.

\section{ACKNOWLEDGMENTS}

We wish to thank Abhay Ashtekar, Laurent Freidel, John Klauder, Karel Kuchař and Thomas Thiemann for comments and discussions. This work was supported in part by the National Science Foundation under grants NSFPHY-9423950, NSF-INT-9811610, NSF-PHY-9407194, research funds of the Pennsylvania State University, the Eberly Family research fund at PSU. JP acknowledges support of the Alfred P. Sloan and John Simon Guggenheim foundations. We acknowledge support of PEDECIBA (Uruguay). RG and JP wish to thank the Institute for Theoretical Physics of the University of California at Santa Barbara and CDB, RG and JG the Center for Gravitational Physics and Geometry at Penn State for hospitality during the completion of this work.

[1] C. Di Bartolo, R. Gambini, J. Griego, J. Pullin "Canonical quantum gravity in the Vassiliev invariants arena: I. Kinematical structure", companion paper.

[2] C. Rovelli, L. Smolin, Nucl. Phys. B442, 593 (1995).

[3] J. Barbero, Phys. Rev. D51, 5507 (1995).

[4] T. Thiemann, Class. Quan. Grav. 15, 839 (1998).

[5] R. Borissov, R. De Pietri, C. Rovelli, Class. Quan. Grav. 14, 2793 (1997).

[6] T. Thiemann, J. Math. Phys. 39, 3347 (1988).

[7] R. Gambini, Phys. Lett. B255, 180 (1991).

[8] R. Gambini, A. Garat, J. Pullin, Int. J. Mod. Phys. D4, 589 (1995).

[9] R. Gambini, J. Pullin "Loops, knots, gauge theories and quantum gravity" Cambridge University Press (1996).

[10] T. Thiemann, Class. Quan. Grav. 15, 1207 (1998).

[11] R. Gambini, J. Lewandowski, D. Marolf, J. Pullin, Int. J. Mod. Phys. D7 97 (1997).

[12] B. Brügmann, R. Gambini, J. Pullin, Phys. Rev. Lett. 68431 (1992).

[13] J. Griego, Phys. Rev. D53 6966 (1996).

[14] R. Gambini, J. Pullin, Phys. Rev. D54 5935 (1996).

[15] C. Rovelli, L. Smolin, Phys. Rev. Lett. 611155 (1988).

[16] J. Lewandowski, D. Marolf, Int. J. Mod. Phys. D7, 299 (1998).

[17] J. Lewandowski, T. Thiemann, Class. Quant. Grav. 162299 (1999).

[18] B. Brügmann, R. Gambini, J. Pullin, Nucl. Phys. B385, 587 (1992).

[19] R. Gambini, J. Griego, J. Pullin, Nucl. Phys. B534, 675-696 (1998).

[20] R. Gambini, J. Pullin, Phys. Rev. D59, 124021 (1999).

[21] T. Thiemann, Class. Quan. Grav. 15, 1249 (1998).

[22] D. Marolf, D. Giulini, Class. Quant. Grav. 162479 (1999).

[23] See for instance A. Ashtekar, J. Baez, A. Corichi, K. Krasnov Phys. Rev. Lett. 80, 904 (1998), and references therein.

[24] L. Freidel, K. Krasnov, R. Puzio, hep-th/9901069. 